\title{
INTÉGRALES STOCHASTIQUES \\ DE PROCESSUS ANTICIPANTS ET PROJECTIONS DUALES PRÉVISIBLES
}

\author{
C. Donati-Martin et M. Yor
}

Abstract

We define a stochastic anticipating integral $\delta^{\mu}$ with respect to Brownian motion, associated to a non adapted increasing process $\left(\mu_{t}\right)$, with dual projection $t$. The integral $\delta^{\mu}(u)$ of an anticipating process $\left(u_{t}\right)$ satisfies: for every bounded predictable process $f_{t}$,

$$
E\left[\left(\int f_{s} d B_{s}\right) \delta^{\mu}(u)\right]=E\left[\int f_{s} u_{s} d \mu_{s}\right] .
$$

We characterize this integral when $\mu_{t}=\sup _{t \leq s<1} B_{s}$. The proof relies on a path decomposition of Brownian motion up to time 1.

\section{Introduction}

Soit $\left(X_{t} ; 0 \leq t \leq 1\right)$ un mouvement brownien réel issu de 0 , et $\left(\mathcal{F}_{t} ; 0 \leq t \leq 1\right)$ sa filtration naturelle. Pour une large classe de processus $\left(u_{t} ; 0 \leq t \leq 1\right)$ mesurables pour $\mathcal{F}_{1} \otimes \mathcal{B}([0,1])$, l'intégrale de Skorohod (par rapport au mouvement brownien $X$ ) du processus $u$, notée $\delta(u)$, est caractérisée par la formule de dualité suivante:

Pour toute variable $F$ de carré intégrable, dérivable au sens de Malliavin,

$$
E(F \delta(u))=E\left(\int_{0}^{1} D_{t} F u_{t} d t\right) .
$$

La formule (1.1) est due à Gaveau et Trauber [6] qui ont identifié l'intégrale $\delta$ définie par Skorohod en [14] comme l'adjoint de l'opérateur de dérivation $D$. Nous renvoyons à Malliavin [8] et Nualart [10] pour la définition de l'opérateur $D$ et les notions de calcul des variations sur l'espace de Wiener.

Keywords. Stochastic anticipating integral, dual projection, path decomposition, balayage formula. 
Nous nous proposons de définir une (ou plusieurs) intégrales stochastiques de processus $\left(u_{t}\right)$ supposés seulement $\mathcal{F}_{1} \otimes \mathcal{B}([0,1])$ mesurables, à partir de la remarque suivante. Considérons dans le terme de droite de (1.1) non pas les termes $d t, D_{t} F$ et $u_{t}$ individuellement, mais plutôt la mesure aléatoire $D_{t} F d t$ d'une part, et le processus $\left(u_{t}\right)$ d'autre part. Le fait que l'intégrale de Skorohod $\delta(u)$ coïncide avec l'intégrale d'Itô que nous noterons ici $\int(u):=\int_{0}^{1} u_{s} d X_{s}$ lorsque $u$ est prévisible, provient de ce que la projection duale prévisible de la mesure $D_{t} F d t$ est $E\left(D_{t} F / \mathcal{F}_{t}\right) d t$, c'est à dire la mesure $f_{t} d t$, où $\left(f_{t}\right)$ est l'intégrand prévisible qui figure dans la représentation d'Itô de $F^{(*)}$, i.e.:

$$
F=E(F)+\int_{0}^{1} f_{t} d X_{t} .
$$

Ainsi, lorsque $u$ est prévisible,

$$
E\left(\int_{0}^{1} D_{t} F u_{t} d t\right)=E\left(\int_{0}^{1} f_{t} u_{t} d t\right)=E\left(F \int(u)\right) .
$$

Cette remarque étant faite, nous pouvons associer à tout processus croissant $\left(\mu_{t} ; 0 \leq t \leq 1\right)$ dont la projection duale prévisible, notée $\mu_{t}^{(p)}$ est $t$, une intégrale stochastique ${ }^{(\mu)} \delta$ définie de la façon suivante:

Définition et Théorème 1.1. A tout processus $\left(u_{t} ; 0 \leq t \leq 1\right)$ tel que

$$
E\left(\int_{0}^{1} u_{s}^{2} d \mu_{s}\right)<\infty
$$

on associe sa projection hilbertienne, notée $u^{\mu}$, sur $L^{2}\left(d \mu_{s} d P ; \mathcal{P}\right)$, où $\mathcal{P}$ désigne la tribu prévisible. Cette projection satisfait:

1. $E\left(\int_{0}^{1}\left(u_{s}^{\mu}\right)^{2} d s\right)<\infty$,

2. pour toute v.a. $F \in L^{2}\left(\mathcal{F}_{1}\right)$,

$$
E\left(F \int\left(u^{\mu}\right)\right)=E\left(\int_{0}^{1} f_{t} u_{t} d \mu_{t}\right)
$$

où $f_{t}$ est l'intégrand prévisible figurant dans la représentation d'Itô de $F$.

Dans la suite, on notera l'intégrale ainsi définie: ${ }^{\mu} \delta(u)=\int\left(u^{\mu}\right)$.

(*) Cette représentation de l'intégrand $f$ associé à $F$ est connue sous le nom de formule de Clark-Ocone [3], [11]. Ocone établit (1.2) avec $f_{t}=E\left(D_{t} F / \mathcal{F}_{t}\right)$ à partir de l'identité $\delta(u)=\int(u)$ pour $u$ processus adapté. 
Par définition même de $u^{\mu}$, l'égalité $u=u^{\mu}$ a lieu dans $L^{2}\left(d \mu_{s} d P ; \mathcal{P}\right)$ si et seulement si $u$ est prévisible. En particulier, ${ }^{\mu} \delta$ et $\int$ coïncident sur l'espace

$$
L^{2}\left(d \mu_{s} d P ; \mathcal{P}\right) \equiv L^{2}(d s d P ; \mathcal{P}) .
$$

Plus généralement, on a, pour tout processus prévisible borné $h$ :

$$
(h u)^{\mu}=h u^{\mu} .
$$

Démonstration du Théorème 1.1: La propriété 1) est immédiate; en ce qui concerne 2), on a: pour tout processus prévisible $f$,

$E\left(\int_{0}^{1} f_{t} u_{t} d \mu_{t}\right)=E\left(\int_{0}^{1} f_{t} u_{t}^{\mu} d \mu_{t}\right)=E\left(\int_{0}^{1} f_{t} u_{t}^{\mu} d t\right)=E\left(F \int\left(u^{\mu}\right)\right)$.

La première égalité provient de la définition de $u^{\mu}$ et la deuxième de l'hypothèse: $\mu_{t}^{(p)}=t$ ( $f u^{\mu}$ est prévisible).

Nous donnons dans la proposition suivante, une première règle très générale de calcul du processus $u^{\mu}$.

Proposition 1.1. Soit $\left(u_{t} ; t \leq 1\right)$ un processus mesurable tel que:

$$
E\left(\int_{0}^{1} u_{t}^{2} d \mu_{t}\right)<\infty
$$

Alors, le processus $Z_{t}^{\mu}(u)=E\left(\int_{0}^{t} u_{s} d \mu_{s} \mid \mathcal{F}_{t}\right)$ est une quasi-martingale, dont on note la décomposition canonique $M_{t}^{\mu}(u)+U_{t}^{\mu}(u), U_{t}^{\mu}(u)$ désignant la partie processus prévisible à variation intégrable. On a alors:

$$
U_{t}^{\mu}(u)=\int_{0}^{t} u_{s}^{\mu} d s
$$

Démonstration: Soit $\tau=\left\{0=t_{0}<t_{1}<t_{2}<\cdots<t_{n}=1\right\}$ une subdivision de $[0,1]$; on note

$$
V_{\tau}\left(Z^{\mu}(u)\right)=\sum_{i=0}^{n-1} E\left(\left|E\left(Z_{t_{i+1}}^{\mu}(u)-Z_{t_{i}}^{\mu}(u) / \mathcal{F}_{t_{i}}\right)\right|\right) .
$$

Alors,

$$
V_{\tau}\left(Z^{\mu}(u)\right) \leq E\left(\int_{0}^{1}\left|u_{s}\right| d \mu_{s}\right) \leq\left(E\left(\int_{0}^{1} u_{s}^{2} d \mu_{s}\right)\right)^{\frac{1}{2}}<\infty,
$$

prouvant la propriété de quasi-martingale de $Z^{\mu}(u)$. 
Soit $\left(h_{s}\right)$ un processus prévisible élémentaire borné de la forme $h_{s}=$ $H 1_{] a, b]}(s)$ avec $H \in \mathcal{F}_{a}$.

$$
\begin{aligned}
E\left(\int_{0}^{1} h_{s} u_{s} d \mu_{s}\right) & =E\left(H \int_{a}^{b} u_{s} d \mu_{s}\right) \\
& =E\left(H\left(Z_{b}^{\mu}(u)-Z_{a}^{\mu}(u)\right)\right) \\
& =E\left(H\left(U_{b}^{\mu}(u)-U_{a}^{\mu}(u)\right)\right) \\
& =E\left(\int_{0}^{1} h_{s} d U_{s}^{\mu}(u)\right) .
\end{aligned}
$$

On obtient donc $\left(u_{s} d \mu_{s}\right)_{s}^{(p)}=d U_{s}^{\mu}(u)$, c'est à dire $u_{s}^{\mu} d s=d U_{s}^{\mu}(u)$.

La Définition 1.1 n'aurait bien entendu aucun intérêt si l'on ne pouvait exhiber des processus croissants "intéressants" $\mu_{t}$ vérifiant $\mu_{t}^{(p)}=t$. Or, de telles constructions ont été faites précisement en [1] par intégration des résultats correspondants pour les temps locaux d'une martingale. De façon précise, on a la

Proposition 1.2 (Azéma-Yor $[\mathbf{1}])$. Soit $\left(M_{t} ; t \geq 0\right)$ une martingale continue, de carré intégrable, dans une filtration $\left(\mathcal{G}_{t} ; t \geq 0\right)$. Alors, le processus croissant $\left(\langle M\rangle_{t} ; t \geq 0\right)$ est la projection duale prévisible de chacun des deux processus croissants (non adaptés):

$$
\begin{aligned}
& \mu_{t}^{(+)}=\left(I_{0}-M_{\infty}\right)^{2}-\left(I_{t}-M_{\infty}\right)^{2} \\
& \mu_{t}^{(-)}=\left(J_{0}-M_{\infty}\right)^{2}-\left(J_{t}-M_{\infty}\right)^{2}
\end{aligned}
$$

ò̀ $I_{t}=\sup _{s \geq t} M_{s}, J_{t}=\inf _{s \geq t} M_{s}$.

Dans cet article, pour rester "concrets", nous appliquerons seulement la proposition ci-dessus à $M_{t}=X_{t \wedge 1}$, avec $\left(X_{t} ; t \geq 0\right)$ mouvement brownien réel; ainsi, on a:

$$
\mu_{t}^{(+)}=\left(I_{0}-X_{1}\right)^{2}-\left(I_{t}-X_{1}\right)^{2} \text { où } I_{t}=\sup _{t \leq s \leq 1} X_{s} .
$$

Nous noterons ${ }^{(+)} \delta$ pour ${ }^{\mu^{(+)}} \delta$ (resp. ${ }^{(-)} \delta$ pour ${ }^{\mu^{(-)}} \delta$ ); ainsi que $u^{(+)}$ $\left(\right.$ resp. $\left.u^{(-)}\right)$la projection de $u \operatorname{sur} L^{2}\left(d \mu_{t}^{(+)} d P ; \mathcal{P}\right)\left(\operatorname{resp} . L^{2}\left(d \mu_{t}^{(-)} d P ; \mathcal{P}\right)\right)$; cette projection étant notée $\Pi^{(+)}$, resp. $\Pi^{(-)}$. 
Le paragraphe 2 est consacré à l'étude des propriétés de l'intégrale ${ }^{(+)} \delta$ en particulier, alors que dans le paragraphe 3 , nous montrons comment cette intégrale peut être désintégrée suivant les temps locaux browniens; dans le même esprit, nous montrons, au paragraphe 4, quelques relations entre notre construction et la formule de balayage. Enfin, au paragraphe 5 , nous considérons un autre exemple d'intégrale ${ }^{\mu} \delta$, très lié au théorème de représentation, dû à J. Pitman, du processus de Bessel de dimension 3 comme $\left(2 S_{t}-X_{t} ; t \geq 0\right)$ où $S_{t}=\sup _{s \leq t} X_{s}$.

\section{Quelques propriétés de l'intégrale ${ }^{\mu} \delta$}

Nous présentons deux propriétés importantes de ${ }^{(+)} \delta$.

Proposition 2.1. Soit $\left(u_{t}\right)$ un processus mesurable tel que

$$
E\left(\int_{0}^{1} u_{s}^{2} d \mu_{s}^{(+)}\right)<\infty
$$

1. Si $\varphi:[0,1] \times \mathbb{R} \rightarrow \mathbb{R}$ est une fonction bornée mesurable, alors:

$$
(\varphi(\cdot, I .) u)^{(+)}=\varphi(\cdot, X .) u^{(+)} .
$$

2. Si u est un processus rétrograde, i.e. $u_{t}$ est $\mathcal{F}^{t}$ adapté, où

$$
\mathcal{F}^{t}=\sigma\left\{X_{s}-X_{1} ; s \geq t\right\},
$$

alors $u^{(+)}$est déterministe.

Démonstration: 1) Pour toute variable $F \in L^{2}(\Omega)$,

$$
E\left(F \int\left((u \varphi(\cdot, I))^{(+)}\right)\right)=E\left(\int_{0}^{1} f_{s} \varphi\left(s, I_{s}\right) u_{s} d \mu_{s}^{(+)}\right) .
$$

Or, la mesure $d \mu_{s}^{(+)}$est portée par $\left\{s ; X_{s}=I_{s}\right\}$; ainsi, le membre de droite de l'équation précédente est égal à $E\left(\int_{0}^{1} f_{s} \varphi\left(s, X_{s}\right) u_{s} d \mu_{s}^{(+)}\right)$. On utilise alors (1.5).

2) D'après la Proposition $1.1, \int_{0}^{t} u_{s}^{(+)} d s$ est la partie à variation intégrable de la quasi-martingale

$$
\begin{aligned}
Z_{t}^{(+)}(u) & =E\left(\int_{0}^{t} u_{s} d \mu_{s}^{(+)} \mid \mathcal{F}_{t}\right) \\
& =E\left(\int_{0}^{1} u_{s} d \mu_{s}^{(+)} \mid \mathcal{F}_{t}\right)-E\left(\int_{t}^{1} u_{s} d \mu_{s}^{(+)} \mid \mathcal{F}_{t}\right) .
\end{aligned}
$$


Or, si $u$ est rétrograde, le processus $\int_{.}^{1} u_{s} d \mu_{s}^{(+)}$est également rétrograde, $\int_{t}^{1} u_{s} d \mu_{s}^{(+)}$est donc indépendant de $\mathcal{F}_{t}$ et

$$
\int_{0}^{t} u_{s}^{(+)} d s=-E\left(\int_{t}^{1} u_{s} d \mu_{s}^{(+)}\right)
$$

Nous allons maintenant appliquer la Proposition 1.1 au calcul de $u^{(+)}$ lorsque $u_{s} \equiv \Phi$ variable aléatoire $\mathcal{F}_{1}$ mesurable, telle que $E\left(\Phi^{2+\varepsilon}\right)<\infty$, pour un $\varepsilon>0$, ce qui assure:

$$
E\left(\Phi^{2} \mu_{1}^{(+)}\right)<\infty .
$$

Dans ce cas, il existe une fonctionnelle $\Phi_{t}\left(\omega, \omega^{\prime}\right)$ qui est mesurable en $\left(t, \omega, \omega^{\prime}\right)$ telle que pour tout $t \leq 1$,

$$
\Phi=\Phi_{t}\left(\left\{X_{s}, s \leq t\right\} ;\left\{X_{s}-X_{1} ; s \geq t\right\}\right) .
$$

On peut alors écrire:

$$
\begin{aligned}
& E\left(\Phi \mu_{t}^{(+)} / \mathcal{F}_{t}\right)=E\left(\Phi\left(I_{0}-X_{1}\right)^{2} / \mathcal{F}_{t}\right) \\
& \quad-E\left(\Phi_{t}\left(\left\{X_{s}, s \leq t\right\} ;\left\{X_{s}-X_{1} ; s \geq t\right\}\right)\left(I_{t}-X_{1}\right)^{2} / \mathcal{F}_{t}\right) .
\end{aligned}
$$

La partie à variation bornée de cette quasi-martingale est celle de la quasi-martingale:

$$
\begin{aligned}
& \Psi_{t}\left(X_{s} ; s \leq t\right) \\
:= & -\hat{E}\left(\Phi_{t}\left(\left\{X_{s}, s \leq t\right\} ;\left\{\hat{X}_{s}-\hat{X}_{1} ; s \geq t\right\}\right)\left(\sup _{s \geq t}\left(\hat{X}_{s}-\hat{X}_{1}\right)\right)^{2}\right),
\end{aligned}
$$

où $X$ et $\hat{X}$ sont deux mouvements browniens indépendants et l'espérance, notée $\hat{E}$ est prise par rapport à $\hat{X}$. Nous donnons maintenant quelques exemples explicites de calculs de la quasi-martingale figurant en (2.1). Nous montrerons par une autre approche que celle développée dans les exemples ci-dessous, la formule générale suivante:

$$
\Pi_{s}^{(+)}\left(\varphi\left(X_{1}\right)\right)=\hat{E}\left(\varphi\left(-\sqrt{1-s} \hat{R}_{1}+X_{s}\right)\right)
$$

où $\hat{R}_{1}$ désigne la valeur à l'instant 1 , d'un processus de Bessel de dimension 3 , indépendant de $X$, l'espérance étant prise par rapport à la loi de $\hat{R}$ (voir Proposition 3.1). 
Exemple 1. $\Phi=X_{1}$.

$$
\begin{aligned}
-E\left(X_{1}\left(I_{t}-X_{1}\right)^{2} / \mathcal{F}_{t}\right) & =-X_{t} E\left(\left(I_{t}-X_{1}\right)^{2}\right)+E\left(\left(X_{t}-X_{1}\right)\left(I_{t}-X_{1}\right)^{2}\right) \\
& =-X_{t} \hat{E}\left(\hat{S}_{1-t}^{2}\right)+\hat{E}\left(\hat{X}_{1-t} \hat{S}_{1-t}^{2}\right) \\
& =-X_{t}(1-t)+\hat{E}\left(\hat{X}_{1-t} \hat{S}_{1-t}^{2}\right)
\end{aligned}
$$

où $\hat{X}_{u}$ est un mouvement brownien et $\hat{S}_{u}=\sup _{s \leq u} \hat{X}_{s}$. Or,

$$
\begin{aligned}
& \hat{E}\left(\hat{X}_{1-t} \hat{S}_{1-t}^{2}\right)=\hat{E}\left(\int_{0}^{1-t} \hat{X}_{u} d\left(\hat{S}_{u}^{2}\right)\right)=2 \hat{E}\left(\int_{0}^{1-t} \hat{S}_{u}^{2} d \hat{S}_{u}\right)=\frac{2}{3} \hat{E}\left(\left(\hat{S}_{1-t}\right)^{3}\right), \\
& \hat{E}\left(\hat{X}_{1-t} \hat{S}_{1-t}^{2}\right)=\frac{2}{3}(1-t)^{\frac{3}{2}} E\left(\left|B_{1}\right|^{3}\right)=\frac{2}{3}(1-t)^{\frac{3}{2}} \frac{2 \sqrt{2}}{\sqrt{\pi}} .
\end{aligned}
$$

Avec les notations de la Proposition 1.1,

$$
U_{t}^{(+)}\left(X_{1}\right)=\int_{0}^{t} X_{s} d s+\frac{4 \sqrt{2}}{3 \sqrt{\pi}}(1-t)^{\frac{3}{2}}
$$

et donc:

$$
u_{s}^{(+)}=\Pi_{s}^{(+)}\left(X_{1}\right)=X_{s}-\frac{2 \sqrt{2}}{\sqrt{\pi}}(1-s)^{\frac{1}{2}} .
$$

Exemple 2. $\Phi=\exp \left(\lambda X_{1}\right)$.

$$
\begin{aligned}
-E\left(\exp \left(\lambda X_{1}\right)\left(I_{t}-X_{1}\right)^{2} / \mathcal{F}_{t}\right) & =-\exp \left(\lambda X_{t}\right) E\left(\exp \left(-\lambda\left(X_{t}-X_{1}\right)\right)\left(I_{t}-X_{1}\right)^{2}\right) \\
& =-\exp \left(\lambda X_{t}\right) \hat{E}\left(\exp \left(-\lambda \hat{X}_{1-t}\right) \hat{S}_{1-t}^{2}\right) .
\end{aligned}
$$

Notons $f_{\lambda}(u)=E\left(\exp \left(-\lambda X_{u}\right) S_{u}^{2}\right)$. La partie à variation bornée de la quasi-martingale précédente est:

$$
\int_{0}^{t}\left(f_{\lambda}^{\prime}(1-s)-\frac{\lambda^{2}}{2} f_{\lambda}(1-s)\right) \exp \left(\lambda X_{s}\right) d s
$$

D'après la formule d'Itô,

$$
\begin{aligned}
\exp \left(-\lambda X_{t}\right) S_{t}^{2}= & -\lambda \int_{0}^{t} \exp \left(-\lambda X_{s}\right) S_{s}^{2} d X_{s} \\
& +2 \int_{0}^{t} \exp \left(-\lambda X_{s}\right) S_{s} d S_{s}+\frac{\lambda^{2}}{2} \int_{0}^{t} \exp \left(-\lambda X_{s}\right) S_{s}^{2} d s
\end{aligned}
$$

et

$$
f_{\lambda}(t)=2 E\left(\int_{0}^{t} \exp \left(-\lambda X_{s}\right) S_{s} d S_{s}\right)+\frac{\lambda^{2}}{2} \int_{0}^{t} f_{\lambda}(s) d s .
$$


Notons $g_{\lambda}(t)=2 E\left(\int_{0}^{t} \exp \left(-\lambda X_{s}\right) S_{s} d S_{s}\right)$; alors, d'après l'égalité précédente, on a:

$$
f_{\lambda}^{\prime}(t)-\frac{\lambda^{2}}{2} f_{\lambda}(t)=g_{\lambda}^{\prime}(t)
$$

Or, par définition de $g_{\lambda}$, on a:

$$
\begin{aligned}
g_{\lambda}(t) & =2 E\left(\int_{0}^{t} \exp \left(-\lambda S_{s}\right) S_{s} d S_{s}\right) \\
& =2 E\left(\exp \left(-\lambda S_{t}\right)\left(\frac{-1}{\lambda} S_{t}-\frac{1}{\lambda^{2}}\right)+\frac{1}{\lambda^{2}}\right) \\
& =2 E\left(\exp \left(-\lambda \sqrt{t} S_{1}\right)\left(\frac{-\sqrt{t}}{\lambda} S_{1}-\frac{1}{\lambda^{2}}\right)+\frac{1}{\lambda^{2}}\right) .
\end{aligned}
$$

En conséquence,

$$
g_{\lambda}^{\prime}(t)=E\left(S_{1}^{2} \exp \left(-\lambda \sqrt{t} S_{1}\right)\right)=E\left(X_{1}^{2} \exp \left(-\lambda \sqrt{t}\left|X_{1}\right|\right)\right)=\varphi(\lambda \sqrt{t}),
$$

où l'on a noté:

$$
\varphi(x)=E\left(X_{1}^{2} \exp \left(-x\left|X_{1}\right|\right)\right)=E\left(\exp \left(-x R_{1}\right)\right)
$$

où $\left(R_{s}, s \geq 0\right)$ désigne un processus de Bessel de dimension 3 issu de 0 . On a ainsi obtenu:

$$
\Pi_{t}^{(+)}\left(\exp \left(\lambda X_{1}\right)\right)=\exp \left(\lambda X_{t}\right) \varphi(\lambda \sqrt{1-t})
$$

où $\varphi$ est définie par la formule (2.2).

Exemple 3. $\Phi=X_{1}^{n}, n \in \mathbb{N}^{*}$.

Soit $\lambda \in \mathbb{R}$,

$$
\Pi^{(+)}\left(\exp \left(\lambda X_{1}\right)\right)=\sum_{n=0}^{\infty} \frac{\lambda^{n}}{n !} \Pi^{(+)}\left(X_{1}^{n}\right) .
$$

Or, d'après l'Exemple 2,

$$
\Pi_{t}^{(+)}\left(\exp \left(\lambda X_{1}\right)\right)=\exp \left(\lambda X_{t}\right) \varphi(\lambda \sqrt{1-t})
$$

avec

$$
\varphi(x)=E\left(\exp \left(-x R_{1}\right)\right)=\sum_{p=0}^{\infty}(-1)^{p} \frac{x^{p}}{p !} \alpha_{p},
$$


où $\alpha_{p}=E\left(R_{1}^{p}\right)$. Ainsi,

$$
\begin{aligned}
\Pi_{t}^{(+)}\left(\exp \left(\lambda X_{1}\right)\right) & =\left(\sum_{k=0}^{\infty} \frac{\lambda^{k}}{k !} X_{t}^{k}\right)\left(\sum_{p=0}^{\infty}(-1)^{p} \frac{(\lambda \sqrt{1-t})^{p}}{p !} \alpha_{p}\right) \\
& =\sum_{n=0}^{\infty} \frac{\lambda^{n}}{n !}\left(\sum_{k=0}^{n}(-1)^{n-k} C_{n}^{k} \alpha_{n-k}(1-t)^{\frac{n-k}{2}} X_{t}^{k}\right) .
\end{aligned}
$$

En identifiant la dernière formule avec le membre de droite dans l'équation (2.4), on obtient:

$$
\text { Pour tout } n, \quad \Pi_{t}^{(+)}\left(X_{1}^{n}\right)=\sum_{k=0}^{n}(-1)^{n-k} C_{n}^{k} \alpha_{n-k}(1-t)^{\frac{n-k}{2}} X_{t}^{k},
$$

avec $\alpha_{p}=E\left(R_{1}^{p}\right)=E\left(\left|X_{1}\right|^{p+2}\right)$. Définissons les polynômes

$$
\tilde{h}_{n}(x)=\sum_{k=0}^{n}(-1)^{n-k} C_{n}^{k} \alpha_{n-k} x^{k}=E\left(\left(x-R_{1}\right)^{n}\right),
$$

et

$$
\tilde{H}_{n}(x, a)=a^{n / 2} \tilde{h}_{n}(x / \sqrt{a}) \text { pour } a>0 ; \quad \tilde{H}_{n}(x, 0)=x^{n} .
$$

La formule (2.5) s'écrit encore:

$$
\Pi_{t}^{(+)}\left(\tilde{H}_{n}\left(X_{1}, 0\right)\right)=\Pi_{t}^{(+)}\left(X_{1}^{n}\right)=\tilde{H}_{n}\left(X_{t}, 1-t\right)
$$

ou encore

$$
{ }^{(+)} \delta\left(\tilde{H}_{n}\left(X_{1}, 0\right)\right)=\int\left(\tilde{H}_{n}(X ., 1-\cdot)\right)
$$

\section{Remarques.}

i) Les polynômes $\tilde{h_{n}}$ introduits en (2.6) peuvent être définis au moyen du développement en série

$$
\exp (\alpha x) \varphi(\alpha)=\sum_{n=0}^{\infty} \frac{\alpha^{n}}{n !} \tilde{h}_{n}(x),
$$

où la fonction $\varphi$ est donnée par (2.2). De plus, cette suite de polynômes satisfait à la relation:

$$
\frac{d}{d x}\left(\tilde{h}_{n}(x)\right)=n \tilde{h}_{n-1}(x), \quad \text { pour tout } n \geq 1 .
$$


ii) On a un résultat analogue à l'Exemple 3 pour l'intégrale de Skorohod, à savoir

$$
\delta\left(H_{n}\left(X_{1}, 1\right)\right)=\int\left(H_{n}\left(X_{.}, \cdot\right)\right)
$$

où $H_{n}(x, a)=a^{n / 2} h_{n}\left(\frac{x}{\sqrt{a}}\right)$ et $\left(h_{n} ; n \geq 0\right)$ désigne la famille des polynômes d'Hermite, définie par le développement en série:

$$
\exp \left(\alpha x-\frac{\alpha^{2}}{2}\right)=\sum_{n=0}^{\infty} \frac{\alpha^{n}}{n !} h_{n}(x)
$$

\section{Désintégration en $a$ des résultats précédents}

Reprenons le résultat obtenu par Azéma et Yor [1] énoncé dans la Proposition 1.2 ci-dessus, dont la preuve est une conséquence du résultat suivant:

La projection duale prévisible du processus croissant non adapté $\mu_{t}^{(a)} \stackrel{\text { def }}{=}\left(X_{1}-a\right)^{+} 1_{\left(g^{a} \leq t\right)}$ est $\frac{1}{2} L_{t}^{a}$, où $L_{t}^{a}$ désigne le temps local en a du mouvement brownien $X$ et $g^{a}=\sup \left\{s \leq 1, X_{s}=a\right\}$.

On a alors $\mu_{t}^{(-)}=2 \int_{\mathbb{R}} d a \mu_{t}^{(a)}$, et de même $\mu_{t}^{(+)}=2 \int_{\mathbb{R}} d a \tilde{\mu}_{t}^{(a)}$ où $\tilde{\mu}_{t}^{(a)}=\left(X_{1}-a\right)^{-} 1_{\left(g^{a} \leq t\right)}$.

Lemme 3.1. Soit $\left(u_{s}\right)$ un processus mesurable vérifiant $E\left(\int_{0}^{1} u_{s}^{2} d \mu_{s}^{(-)}\right)<\infty$. Il existe un processus $u_{s}^{(a)}$ qui est $\mathcal{B}(\mathbb{R}) \otimes \mathcal{P}$ mesurable et tel que pour tout $a, u^{(a)}$ soit la projection (notée $\Pi^{(a)}$ ) de $u$ sur

$$
L^{2}\left(\Omega \times[0,1] ; \mathcal{P} ; d \mu_{t}^{(a)} d P\right)=L^{2}\left(\Omega \times[0,1] ; \mathcal{P} ; \frac{1}{2} d L_{t}^{a} d P\right) .
$$

En conséquence, le processus $v_{s}=u_{s}^{\left(X_{s}\right)}$ est un représentant de $u_{s}^{(a)}$ pour tout a et donc de $u_{s}^{(-)}$.

Preuve: Considérons les mesures $m_{a}$ et $\nu_{a}$ sur l'espace mesurable séparable $(\Omega \times[0,1], \mathcal{P})$ définies par

$$
\begin{gathered}
m_{a}(f)=E\left(\int_{0}^{1} f_{s} u_{s} d \mu_{s}^{a}\right), \\
\nu_{a}(f)=\frac{1}{2} E\left(\int_{0}^{1} f_{s} d L_{s}^{a}\right) .
\end{gathered}
$$


$\left(m_{a}\right)_{a}$ et $\left(\nu_{a}\right)_{a}$ sont deux familles mesurables de mesures bornées, telles que pour tout $a, m_{a}$ est absolument continue par rapport à $\nu_{a}$. D'après un théorème de $\operatorname{Doob}([4$, Chap. V $]$ ), il existe un processus $u_{s}^{(a)}(\omega) \mathcal{B}(\mathbb{R}) \otimes \mathcal{P}$ mesurable tel que pour tout $a, u^{(a)}$ soit une densité de $m_{a}$ par rapport à $\nu_{a}$. De plus,

$$
\begin{aligned}
2 E\left[\int_{0}^{1} f_{s} u_{s} d \mu_{s}^{(a)}\right] & =E\left[\int_{0}^{1} f_{s} u_{s}^{(a)} d L_{s}^{a}\right] \\
& =E\left[\int_{0}^{1} f_{s} u_{s}^{\left(X_{s}\right)} d L_{s}^{a}\right]
\end{aligned}
$$

Ainsi, il suffit de calculer $u^{(a)}$ pour obtenir le processus $u^{(-)}$(qui est égal à $u^{X} \cdot$. .

Lemme 3.2. Soit $\left(u_{s} ; s \leq 1\right)$ un processus défini sur l'espace canonique $\Omega=C([0,1], \mathbb{R})$ qui est $\mathcal{B}[0,1] \otimes \mathcal{F}_{1}$ mesurable et tel que $E\left(\int_{0}^{1} u_{s}^{2} d \mu_{s}\right)<\infty$. Il existe une fonctionnelle $F$ sur $\Omega \times \mathcal{B}[0,1] \times \Omega$ qui est $\mathcal{P} \otimes \mathcal{F}_{1}$ mesurable tel que:

$$
u_{t}(\omega)=F\left(\omega ; t ; \theta_{t}(\omega)\right)
$$

où $\theta_{t}$ est l'opérateur de translation sur $\Omega$ défini par:

$$
\theta_{t}(\omega)_{s}=\omega_{(t+s)}-\omega_{t}, \quad s \leq 1-t .
$$

On introduira aussi la fonctionnelle $\tilde{F}$ vérifiant:

$$
u_{t}(\omega)=\tilde{F}\left(\omega ; t ; \tilde{\theta}_{t}(\omega)\right)
$$

où $\tilde{\theta}_{t}$ est défini par:

$$
\tilde{\theta}_{t}(\omega)_{s}=\frac{\omega(t+(1-t) s)-\omega(t)}{\sqrt{1-t}} .
$$

Preuve: Il suffit de poser $F(\omega ; t ; \hat{\omega})=u_{t}(\omega / t / \hat{\omega})$ où $\omega / t / \hat{\omega}$ est l'élément de $\Omega$ défini par:

$$
\begin{aligned}
(\omega / t / \hat{\omega})(s) & =\omega(s) & & \text { si } s \leq t \\
& =\omega(t)+\hat{\omega}(s-t) & & \text { si } s \geq t .
\end{aligned}
$$

On a $\omega / t / \theta_{t}(\omega)=\omega$, d'où la représentation (3.2).

$((t, \omega) ; \hat{\omega}) \rightarrow u_{t}(\omega / t / \hat{\omega})$ est $\mathcal{P} \otimes \mathcal{F}_{1}$ mesurable (cf $[\mathbf{9}$, Théorème 2$\left.]\right)$.

Nous pouvons maintenant énoncer le résultat principal: 
Théorème 3.1. Soit $\left(u_{s} ; s \leq 1\right)$ un processus qui est $\mathcal{B}[0,1] \otimes \mathcal{F}_{1}$ mesurable et tel que $E\left(\int_{0}^{1} u_{s}^{2} d \mu_{s}\right)<\infty$. Soit $F$ la fonctionnelle associée à u par la représentation (3.2). Alors, pour tout a,

$$
\Pi_{t}^{(a)}(u)=\Pi_{t}^{(-)}(u)=\hat{E}\left(F\left(\left\{X_{s} ; s \leq t\right\} ; t ;\left\{\hat{R}_{s} ; s \leq 1-t\right\}\right)\right)
$$

où $\left(\hat{R}_{s} ; s \leq 1\right)$ désigne un processus de Bessel de dimension 3 , l'espérance étant prise par rapport au processus $\hat{R}$. De façon équivalente,

$$
\Pi_{t}^{(a)}(u)=\Pi_{t}^{(-)}(u)=\hat{E}\left(\tilde{F}\left(\left\{X_{s} ; s \leq t\right\} ; t ;\left\{\hat{R}_{s} ; s \leq 1\right\}\right)\right)
$$

où $\tilde{F}$ satisfait (3.3).

Avant de démontrer ce résultat, donnons quelques exemples de représentation (3.2); dans les deux premiers exemples, le processus $u$ est constant en temps, égal à une variable $\Phi \mathcal{F}_{1}$ mesurable.

- Si $\Phi=\varphi\left(X_{1}\right)$, alors

$$
F(\omega ; t ; \hat{\omega})=\varphi\left(\omega_{t}+\hat{\omega}_{1-t}\right) .
$$

- Si $\Phi=\exp \left(\int_{0}^{1} h(s) d X_{s}\right), h \in L^{2}([0,1])$, alors,

$$
F(\omega ; t ; \hat{\omega})=\exp \left(\int_{0}^{t} h(s) d \omega_{s}\right) \exp \left(\int_{0}^{1-t} h(t+s) d \hat{\omega}_{s}\right)
$$

- $u_{t}=1_{(t<g)}$,

$$
F(\omega ; t ; \hat{\omega})=1_{\left(T_{-\omega(t)}(\hat{\omega}) \leq 1-t\right)}
$$

où $T_{x}(\hat{\omega})=\inf \{s>0 ; \hat{\omega}(s)=x\}$.

Preuve de la formule (3.4): Elle s'appuie sur la décomposition de la trajectoire brownienne en $g=\sup \left\{s \leq 1, X_{s}=0\right\}$ :

$$
\left(m_{s}=\frac{1}{\sqrt{1-g}}\left|X_{g+(1-g) s}\right|, s \leq 1\right) \text { est un méandre brownien indé- }
$$
pendant de $\left(X_{s}, s \leq g\right)$, et de $\operatorname{sgn}\left(X_{1}\right)$.

Nous avons besoin d'un résultat similaire lorsqu'on décompose $X$ en $g^{a}=\sup \left\{0 \leq t \leq 1, X_{t}=a\right\}$, où par convention $\sup \emptyset=0$. 
Lemme 3.3. Soit $G$ et $H$ deux fonctionnelles bornées et $\varphi$ une fonction bornée, alors,

$$
\begin{gathered}
E\left(1_{\left(g^{a}>0\right)} G\left(X_{s} ; s \leq g^{a}\right) H\left(\frac{1}{\sqrt{1-g^{a}}}\left|X_{g^{a}+\left(1-g^{a}\right) s}-X_{g^{a}}\right| ; s \leq 1\right) \varphi\left(\operatorname{sgn}\left(X_{1}-a\right)\right)\right) \\
=E\left(1_{\left(g^{a}>0\right)} G\left(X_{s} ; s \leq g^{a}\right)\right) E\left(H\left(m_{s} ; s \leq 1\right)\right) E\left(\varphi\left(\operatorname{sgn}\left(X_{1}-a\right)\right)\right)
\end{gathered}
$$

où $m$ est un méandre brownien.

Preuve: On applique la propriété de Markov forte au temps $T_{a}=\inf \left\{t \leq 1, X_{t}=a\right\}$.

$\operatorname{Sur}\left(g^{a}>0\right)=\left(T_{a}<1\right), T_{a} \leq g^{a}$ p.s., et $g^{a}=T_{a}+\left(1-T_{a}\right) \hat{g}$ où $\hat{g}=\sup \left\{s \leq 1, \hat{X}_{s}=0\right\}$ et $\hat{X}$ est le mouvement brownien, issu de 0 , défini par:

$$
\hat{X}_{s}=\frac{1}{\sqrt{1-T_{a}}}\left(X_{T_{a}+\left(1-T_{a}\right) s}-a\right) .
$$

$\hat{X}$ est indépendant de $T_{a}$ et $\left(X_{s}, s \leq T_{a}\right)$. On utilise ensuite la décomposition de $\hat{X}$ en $\hat{g}$.

Soit $\left(f_{s}\right)$ un processus prévisible,

$$
\begin{aligned}
E\left(\int_{0}^{1} f_{s} u_{s} d \mu_{s}^{(a)}\right)= & E\left(f_{g^{a}} u_{g^{a}}\left(X_{1}-a\right)^{+} 1_{\left(g^{a}>0\right)}\right) \\
= & E\left(f_{g^{a}} \tilde{F}\left(\left\{X_{s} ; s \leq g^{a}\right\} ; g^{a} ;\left\{\hat{X}_{s}^{\left(g^{a}\right)} ; s \leq 1\right\}\right) \hat{X}_{1}^{\left(g^{a}\right)}\right. \\
& \left.\ldots \sqrt{1-g^{a}} 1_{\left(X_{1}>a\right)} 1_{\left(g^{a}>0\right)}\right)
\end{aligned}
$$

où

$$
\hat{X}_{s}^{\left(g^{a}\right)}=\frac{1}{\sqrt{1-g^{a}}}\left(X_{g^{a}+\left(1-g^{a}\right) s}-X_{g^{a}}\right)=\operatorname{sgn}\left(X_{1}-a\right) m_{s} .
$$

D'après le lemme, $\left(m_{s}, s \leq 1\right)$ est un méandre brownien indépendant de $\mathcal{F}_{g^{a}}$. On a donc

$$
\begin{aligned}
& E\left(\int_{0}^{1} f_{s} u_{s} d \mu_{s}^{(a)}\right) \\
= & \frac{1}{2} \int M(d x) E\left(1_{\left(g^{a}>0\right)} f_{g^{a}} \tilde{F}\left(\left\{X_{s} ; s \leq g^{a}\right\} ; g^{a} ;\left\{x_{s} ; s \leq 1\right\}\right) x_{1} \sqrt{1-g^{a}}\right),
\end{aligned}
$$

où $M$ désigne la loi du méandre sur $C\left([0,1], \mathbb{R}_{+}\right)$. 
Soit $x$ une trajectoire fixée de $C\left([0,1], \mathbb{R}^{+}\right)$, on a alors:

$$
\begin{aligned}
E & \left(1_{\left(g^{a}>0\right)} f_{g^{a}} \tilde{F}\left(\left\{X_{s} ; s \leq g^{a}\right\} ; g^{a} ;\left\{x_{s} ; s \leq 1\right\}\right) x_{1} \sqrt{1-g^{a}}\right) \\
& =\frac{2}{E\left(m_{1}\right)} E\left(1_{\left(g^{a}>0\right)} f_{g^{a}} \tilde{F}\left(\left\{X_{s} ; s \leq g^{a}\right\} ; g^{a} ;\left\{x_{s} ; s \leq 1\right\}\right) x_{1}\left(X_{1}-a\right)^{+}\right) \\
& =\frac{1}{E\left(m_{1}\right)} E\left(\int_{0}^{1} d L_{u}^{a} f_{u} \tilde{F}\left(\left\{X_{s} ; s \leq u\right\} ; u ;\left\{x_{s} ; s \leq 1\right\}\right) x_{1}\right),
\end{aligned}
$$

avec $\frac{1}{E\left(m_{1}\right)}=\sqrt{\frac{2}{\pi}}$. La dernière égalité découle de la propriété $\left(\mu_{t}^{(a)}\right)^{(p)}=$ $\frac{1}{2} L_{t}^{a}$. Ainsi,

$$
\begin{aligned}
& E\left(\int_{0}^{1} f_{s} u_{s} d \mu_{s}^{(a)}\right) \\
& =\frac{K}{2} E\left(\int_{0}^{1} d L_{u}^{a} f_{u} \int M(d x) \tilde{F}\left(\left\{X_{s} ; s \leq u\right\} ; u ;\left\{x_{s} ; s \leq 1\right\}\right) x_{1}\right) \\
& =\frac{1}{2} E\left\{\int_{0}^{1} d L_{u}^{a} f_{u} \hat{E}\left(\tilde{F}\left(\left\{X_{s} ; s \leq u\right\} ; u ;\left\{\hat{R}_{s} ; s \leq 1\right\}\right)\right)\right\},
\end{aligned}
$$

d'après la relation d'Imhof $[\mathbf{7}]$ :

$$
\left.P_{0}^{(3)}\right|_{\mathcal{F}_{1}}=\sqrt{\frac{2}{\pi}} X_{1} \cdot M
$$

explicitant la relation d'absolue continuité entre le processus de Bessel de dimension 3 et la loi du méandre. Ceci entraîne (3.5). La relation (3.4) se déduit de (3.5) par propriété de changement d'échelle du processus de Bessel.

\section{Relation avec les formules de balayage}

\subsection{Notations.}

Dans le paragraphe précédent, nous avons explicité l'opérateur de projection $\Pi^{(a)}$. Nous allons maintenant considérer une famille de projecteurs, indexée par le temps, définie de la façon suivante: pour tout $s \in[0,1]$ et $a \in \mathbb{R}$, on note $\Pi^{(a), s}$ le projecteur de

$$
L^{2}\left(\Omega \times[0, s] ; \mathcal{F}_{s} \otimes \mathcal{B}[0, s] ; d \mu_{t}^{(a), s} d P\right)
$$


sur

$$
L^{2}\left(\Omega \times[0, s] ; \mathcal{P}_{s} ; \frac{1}{2} d L_{t}^{a} 1_{(t \leq s)} d P\right),
$$

où

$$
\left\{\begin{array}{l}
\mu_{t}^{(a), s} \stackrel{\text { def }}{=}\left(X_{s}-a\right)^{+} 1_{\left(g_{s}^{a} \leq t\right)}, \quad t \leq s ; \\
g_{s}^{a}=\sup \left\{t \leq s, X_{t}=a\right\} .
\end{array}\right.
$$

$\mathcal{P}_{s}$ désigne la tribu prévisible sur $\Omega \times[0, s]$.

Ainsi, la projection $\Pi^{(a)}$ définie en Section 3 est $\Pi^{(a), 1}$.

Si $\Phi_{s}$ est une variable $\mathcal{F}_{s}$ mesurable, nous notons:

$$
\tilde{\Pi}_{t}^{(a), s}\left(\Phi_{s}\right)=\Pi_{t}^{(a), s}\left(u^{(s)}\right), \quad t \leq s,
$$

où $u^{(s)}$ est le processus constant en temps: $u_{t}^{(s)}=\Phi_{s}, t \leq s$. $\tilde{\Pi}^{(a), s}$ est une application linéaire continue de

$$
L^{2+\varepsilon}\left(\Omega ; \mathcal{F}_{s} ; d P\right) \rightarrow L^{2}\left(\Omega \times[0, s] ; \mathcal{P}_{s} ; \frac{1}{2} d L_{t}^{a} 1_{(t \leq s)} d P\right)
$$

\subsection{Formule de balayage.}

Soit $Z_{t}=M_{t}+V_{t}$ une semi-martingale continue s'annulant sur $H_{a}=\left\{t \leq 1 ; X_{t}=a\right\}$. Pour tout processus prévisible borné $\left(f_{s}\right)$, nul en 0 , on a $([\mathbf{1 5}])$ :

$$
\left(Z_{u} f_{g_{u}^{a}}-\int_{0}^{u} f_{g_{s}^{a}} d V_{s} ; 0 \leq u \leq 1\right)
$$

est une martingale locale, nulle en 0 . Si, de plus, $Z$ appartient à $H^{1}$, on a:

$$
E\left(Z_{u} f_{g_{u}^{a}}\right)=E\left(\int_{0}^{u} f_{g_{s}^{a}} d V_{s}\right)
$$

Rappelons que $H^{p}$ désigne l'espace des semimartingales continues $\left(Z_{t} ; t \leq 1\right)$ de décomposition canonique $Z=M+V$ vérifiant $\|\langle M\rangle_{1}^{1 / 2}+$ $\int_{0}^{1}\left|d V_{s}\right| \|_{p}<\infty$, si $1 \leq p<\infty$. 


\subsection{Relation différentielle liant les opérateurs $\tilde{\Pi}$.}

Soit $\Phi$ une variable $\mathcal{F}_{1}$ mesurable telle que $E\left(\Phi^{p}\right)<\infty$ pour un $p>2$ et $\Phi_{t}$ une semi-martingale continue, appartenant à $H^{p}$ de variable terminale $\Phi$ (par exemple, on peut prendre $\left.E\left(\phi / \mathcal{F}_{t}\right)\right) . Z_{t}=\Phi_{t}\left(X_{t}-a\right)^{+}$ satisfait les hypothèses d'intégrabilité du paragraphe 4.2 ; ainsi, pour tout processus prévisible $f$,

$$
E\left(\Phi\left(X_{1}-a\right)^{+} f_{g^{a}}\right)=E\left(\int_{0}^{1} f_{g_{s}^{a}} d V_{s}\right),
$$

où, si $\Phi_{t}=M_{t}+C_{t} \equiv \int_{0}^{t} \varphi_{s} d X_{s}+C_{t}$, avec $\left(C_{t}\right)$ processus continu à variation bornée, est la décomposition canonique de $\left(\Phi_{t}\right)$, on obtient:

$$
d V_{s}=\frac{1}{2} \Phi_{s} d L_{s}^{a}+\left(X_{s}-a\right)^{+} d C_{s}+1_{\left(X_{s}>a\right)} \varphi_{s} d s .
$$

On suppose dorénavant: $C_{t}=\int_{0}^{t} c_{s} d s$, et on écrit:

$$
d V_{s}=\frac{1}{2} \Phi_{s} d L_{s}^{a}+\left(X_{s}-a\right)^{+} \eta_{s} d s,
$$

où

$$
\eta_{s}=c_{s}+1_{\left(X_{s}>a\right)} \frac{\varphi_{s}}{X_{s}-a} .
$$

Alors, on a:

$$
\begin{aligned}
& E\left(\Phi\left(X_{1}-a\right)^{+} f_{g^{a}}\right) \\
& \quad=\frac{1}{2} E\left(\int_{0}^{1} f_{s} \Phi_{s} d L_{s}^{a}\right)+\int_{0}^{1} d s E\left(f_{g_{s}^{a}} \eta_{s}\left(X_{s}-a\right)^{+}\right) .
\end{aligned}
$$

Par définition des projecteurs $\tilde{\Pi}^{(a), s}$, on a les relations:

$$
E\left(\Phi\left(X_{1}-a\right)^{+} f_{g^{a}}\right)=\frac{1}{2} E\left(\int_{0}^{1} d L_{t}^{a} f_{t} \tilde{\Pi}_{t}^{(a), 1}(\Phi)\right)
$$

et

$$
E\left(\eta_{s}\left(X_{s}-a\right)^{+} f_{g_{s}^{a}}\right)=\frac{1}{2} E\left(\int_{0}^{s} d L_{t}^{a} f_{t} \tilde{\Pi}_{t}^{(a), s}\left(\eta_{s}\right)\right) .
$$

En combinant les relations (4.5), (4.6) et (4.7), nous obtenons la relation entre les opérateurs $\tilde{\Pi}$ :

$$
\tilde{\Pi}_{t}^{(a), 1}(\Phi)=\Phi_{t}+\int_{t}^{1} \tilde{\Pi}_{t}^{(a), s}\left(\eta_{s}\right) d s \quad d L_{t}^{a} d P \text { p.p. }
$$


ou, plus généralement:

(4.9) $\tilde{\Pi}_{t}^{(a), u}\left(\Phi_{u}\right)=\Phi_{t}+\int_{t}^{u} \tilde{\Pi}_{t}^{(a), s}\left(\eta_{s}\right) d s \quad d L_{t}^{a} d P$ p.p., pour $t \leq u \leq 1$.

Pour mieux comprendre la relation (4.8), considérons les deux exemples suivants:

i) $\Phi=\phi\left(X_{1}\right)$ où $\phi$ est une fonction de classe $C^{2}$,

ii) $\Phi=\exp \left(\int_{0}^{1} h(u) d X_{u}\right), h \in L^{2}([0,1])$.

Choisissons alors $\Phi_{t}$ de la façon suivante:

i) $\Phi_{t}=\phi\left(X_{t}\right)$,

ii) $\Phi_{t}=\exp \left(\int_{0}^{t} h(u) d X_{u}\right)$.

On obtient, d'après (4.4):

i) $\eta_{s}=\frac{1}{2} \phi^{\prime \prime}\left(X_{s}\right)+\frac{\phi^{\prime}\left(X_{s}\right)}{X_{s}-a} 1_{\left(X_{s}>a\right)} \stackrel{\text { def }}{=} \psi\left(X_{s}\right)$,

ii) $\eta_{s}=\frac{1}{2} \Phi_{s} h^{2}(s)+\Phi_{s} \frac{h(s)}{X_{s}-a} 1_{\left(X_{s}>a\right)}$.

D'après le Théorème 3.1, formule (3.4), on a:

i) $\tilde{\Pi}_{t}^{(a), 1}(\Phi)=\hat{E}\left(\phi\left(B_{t}(\omega)+\hat{R}_{1-t}\right)\right)=\hat{E}\left(\phi\left(a+\hat{R}_{1-t}\right)\right) d L_{t}^{a} d P$ p.p., $\tilde{\Pi}_{t}^{(a), s}\left(\eta_{s}\right)=\hat{E}\left(\psi\left(B_{t}(\omega)+\hat{R}_{1-t}\right)\right)=\hat{E}\left(\psi\left(a+\hat{R}_{1-t}\right)\right) d L_{t}^{a} d P$ p.p.

ii) $\tilde{\Pi}_{t}^{(a), 1}(\Phi)=\Phi_{t} \hat{E}\left(\exp \left(\int_{0}^{1-t} h(t+u) d \hat{R}_{u}\right)\right)$,

$$
\begin{aligned}
& \tilde{\Pi}_{t}^{(a), s}\left(\eta_{s}\right)=\frac{1}{2} \Phi_{t} \hat{E}\left(\exp \left(\int_{0}^{s-t} h(t+u) d \hat{R}_{u}\right)\right)+h(s) \Phi_{t} \ldots \\
& \ldots \hat{E}\left(\frac{1}{\hat{R}_{s-t}} \exp \left(\int_{0}^{s-t} h(t+u) d \hat{R}_{u}\right)\right) .
\end{aligned}
$$

Dans les deux cas, on vérifie aisément la formule (4.8) après application de la formule d'Itô au processus $\phi\left(a+\hat{R}_{u}\right)$, resp. $\exp \left(\int_{0}^{u} h(t+v) d \hat{R}_{v}\right)$. On prend ensuite l'espérance des deux membres, dans la formule ainsi obtenue, considérée au temps $u=1-t$. 


\section{Un exemple d'intégrale anticipante lié au processus de Bessel}

Soit $\left(\beta_{t} ; t \geq 0\right)$ un mouvement brownien réel et $R$ le processus de Bessel de dimension 3, solution de

$$
R_{t}=\beta_{t}+\int_{0}^{t} \frac{d s}{R_{s}} .
$$

D'après le théorème de Pitman [12], si l'on note $J_{t}=\inf _{s \geq t} R_{s}$,

$$
2 J_{t}-R_{t}=B_{t},
$$

où $B$ est un mouvement brownien réel. (5.2) s'écrit encore:

$$
R_{t}=2 S_{t}-B_{t},
$$

où $S_{t}=\sup _{s \leq t} B_{s} \equiv J_{t}$. Notons $\left(\mathcal{R}_{t}\right)$, respectivement $\left(\mathcal{B}_{t}\right)$, la filtration naturelle de $\bar{R}$, resp. $B$ et insistons sur l'inclusion stricte: $\left(\mathcal{R}_{t}\right) \nsubseteq\left(\mathcal{B}_{t}\right)$.

Nous pouvons appliquer les résultats de la Section 1 au processus croissant $\mu_{t}=J_{t}^{2}$ dont la projection duale prévisible (sur la petite filtration $\left.\left(\mathcal{R}_{t}\right)\right)$ est $t$. Ainsi, à tout processus $u\left(\mathcal{B}_{t}\right)$ adapté vérifiant:

$$
E\left(\int_{0}^{\infty} u_{s}^{2} d\left(J_{s}^{2}\right)\right)<\infty
$$

on peut associer l'intégrale stochastique anticipante par rapport au mouvement brownien $\beta$, notée $\delta_{J}(u)$ qui vérifie:

$$
E\left(\left(\int_{0}^{\infty} f_{s} d \beta_{s}\right) \delta_{J}(u)\right)=E\left(\int_{0}^{\infty} f_{s} u_{s} d\left(J_{s}^{2}\right)\right)
$$

pour tout processus $\left(f_{s}\right) \mathcal{R}$-prévisible.

Dans ce cas, il est facile de calculer le processus $u^{(\mu)}$ associé à $u$ par la Définition 1.1. En effet, il existe une fonctionnelle mesurable $\Phi$ telle que

$$
u_{s}=\Phi\left(\left\{R_{u} ; u \leq s\right\} ; J_{s}\right)
$$

alors

$$
u_{s}^{(\mu)}=\Phi\left(\left\{R_{u} ; u \leq s\right\} ; R_{s}\right)
$$

en utilisant le même argument qu'en 1) de la Proposition 2.1.

\section{Exemple.}

$$
\delta_{J}\left(B 1_{[0, t]}\right)=\delta_{J}\left(S 1_{[0, t]}\right)=\int_{0}^{t} R_{s} d \beta_{s}
$$

( $B$ et $S$ ont la même projection, à savoir $R$.) 


\section{Quelques remarques.}

1) L'intégrale stochastique $\int_{0}^{t} u_{s} d \beta_{s}$ a un sens comme intégrale d'Itô dans la filtration $\mathcal{B}$, puisque $\beta$ est une $\mathcal{B}$-semimartingale de décomposition

$$
d \beta_{t}=-d B_{t}+2 d S_{t}-\frac{d t}{R_{t}} .
$$

L'intégrale anticipante et l'intégrale d'Itô dans la filtration grossie ne coïncident pas.

2) L'intégrale ainsi définie $\delta_{J}\left(u 1_{[0, t]}\right)$ est une $\mathcal{R}$ martingale. Or, une autre martingale liée au processus croissant $\mu$ apparaît naturellement, à savoir:

$$
M_{t}(u)={ }^{p}\left(\int_{0}^{t} u_{s} d \mu_{s}\right)-\left(\int_{0}^{t} u_{s} d \mu_{s}\right)^{(p)} ; \quad \mu_{s}=J_{s}^{2}
$$

où ${ }^{p} X$ désigne la projection prévisible du processus $X$ par rapport à la filtration $\mathcal{R}$.

\section{Quelques calculs explicites.}

a) $u \equiv 1$,

$$
M_{t}(1)=E\left(J_{t}^{2} / \mathcal{R}_{t}\right)-t=\frac{1}{3} R_{t}^{2}-t=\frac{2}{3} \int_{0}^{t} R_{s} d \beta_{s} .
$$

b) Remarquons que $M_{t}(u)=M_{t}\left(u^{(\mu)}\right)$ où $u$ et $u^{(\mu)}$ sont reliés par les formules (5.4) et (5.5).

Nous allons calculer $M_{t}(u)$ lorsque $u_{s}^{(\mu)}$ ne dépend que de $R_{s}$, i.e. $u_{s}^{(\mu)}=\varphi\left(R_{s}\right)$.

$$
\begin{aligned}
E\left(\int_{0}^{t} u_{s} d \mu_{s} \mid \mathcal{F}_{t}\right) & =2 E\left(\int_{0}^{t} \varphi\left(R_{s}\right) R_{s} d J_{s} \mid \mathcal{F}_{t}\right) \\
& =2 \int_{0}^{t} \varphi\left(R_{s}\right) R_{s} d_{s} E\left(J_{s} \mid \mathcal{F}_{t}\right) .
\end{aligned}
$$

Or, un calcul simple montre que pour $s \leq t$,

$$
E\left(J_{s} \mid \mathcal{F}_{t}\right)=J_{s}^{t}-\frac{1}{2} \frac{\left(J_{s}^{t}\right)^{2}}{R_{t}}
$$




$$
\begin{aligned}
\text { où } J_{s}^{t}=\inf _{s \leq u \leq t} R_{u} . & \\
E\left(\int_{0}^{t} u_{s} d \mu_{s} \mid \mathcal{F}_{t}\right) & =2 \int_{0}^{t} \varphi\left(R_{s}\right) R_{s}\left(d J_{s}^{t}-\frac{J_{s}^{t}}{R_{t}} d J_{s}^{t}\right) \\
& =2 \int_{0}^{t} \varphi\left(J_{s}^{t}\right) J_{s}^{t}\left(d J_{s}^{t}-\frac{J_{s}^{t}}{R_{t}} d J_{s}^{t}\right) \\
& =2\left(\int_{0}^{R_{t}} \varphi(x) x d x-\frac{1}{R_{t}} \int_{0}^{R_{t}} \varphi(x) x^{2} d x\right) \\
& =\Phi\left(R_{t}\right)
\end{aligned}
$$

où $\Phi(z)=2\left(\int_{0}^{z} \varphi(x) x d x-\frac{1}{z} \int_{0}^{z} \varphi(x) x^{2} d x\right)$. Finalement, on obtient:

$$
M_{t}(\varphi(R))=\int_{0}^{t} \Phi^{\prime}\left(R_{s}\right) d \beta_{s}
$$

$\operatorname{avec} \Phi^{\prime}(z)=\frac{2}{z^{2}} \int_{0}^{z} \varphi(x) x^{2} d x$.

\section{Exemple.}

i) $\varphi \equiv 1$, on retrouve $M_{t}(1)=\frac{2}{3} \int_{0}^{t} R_{s} d \beta_{s}$.

ii) $\varphi(x)=x, \Phi^{\prime}(z)=\frac{z^{2}}{2}$ d'où

$$
M_{t}(B)=M_{t}(S)=\frac{1}{2} \int_{0}^{t} R_{s}^{2} d \beta_{s}
$$

\section{Références}

1. J. Azéma et M. Yor, En guise d'introduction, Astérisque 52-53, Temps Locaux (1978), 3-16.

2. J. AzÉma et M. Yor, Sur les zéros des martingales continues, Sém. Prob. XXVI, Lecture Notes in Math. 1526, Springer (1992), 248-306.

3. J. M. C. Clark, The representation of functionals of Brownian motion by stochastic integrals, Ann. Math. Statist. 41 (1970), 12821295. 
4. C. Dellacherie et P. A. Meyer, "Probabilités et potentiel," vol. II, Hermann, Paris, 1980.

5. R. T. Durrett et D. L. Iglehart, Functionals of Brownian meander and Brownian excursion, Ann. Probab. 5 (1977), 130-135.

6. B. Gaveau et P. Trauber, L'intégrale stochastique comme opérateur de divergence dans l'espace fonctionnel, J. Funct. Anal. 46 (1982), 230-328.

7. J. P. Iмноғ, Density factorization for brownian motion and the three-dimensional Bessel processes and applications, J. Appl. Probab. 21 (1984), 500-510.

8. P. Malliavin, "Stochastic Analysis," Springer, Berlin, 1997.

9. P. A. Meyer, La théorie de la prédiction de F. Knight, Sém. Prob. X, Lecture Notes in Math. 511, Springer (1976), 86-103.

10. D. Nualart, "Malliavin calculus and Related Topics," SpringerVerlag, 1995.

11. D. Ocone, Malliavin's Calculus and Stochastic Integral Representations of Functionals of Diffusion Processes, Stochastics Stochastics Rep. 12 (1984), 161-185.

12. J. Pitman, One-dimensional Brownian motion and the three-dimensional Bessel process, Adv. in Appl. Probab. 7 (1975), 511-526.

13. D. Revuz et M. YoR, "Continuous Martingales and Brownian motion," 2nd edition, Springer, Berlin, 1994.

14. A. V. Skorohod, On a generalization of a stochastic integral, Theory Probab. Appl. 20 (1975), 219-233.

15. M. Yor, Sur le balayage des semi-martingales continues, Sém. Prob. XIII, Lecture Notes in Math. 721, Springer (1979), 453-471.

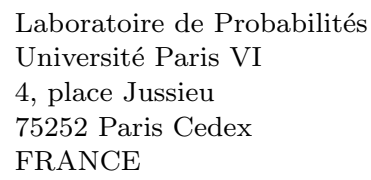

Primera versió rebuda el 28 de juliol de 1998, darrera versió rebuda el 13 de novembre de 1998 